EPJ manuscript No.

(will be inserted by the editor)

\title{
Topological aspects of graphene
}

\section{Dirac fermions and the bulk-edge correspondence in magnetic fields}

\author{
Y. Hatsugai ${ }^{1}$, T. Fukui ${ }^{2}$, and H. Aoki ${ }^{3}$ \\ 1 Department of Applied Physics, University of Tokyo, Hongo, Bunkyo-ku, Tokyo 113, Japan \\ 2 Department of Mathematical Sciences, Ibaraki University, Mito 310-8512, Japan \\ 3 Department of Physics, University of Tokyo, Hongo, Bunkyo-ku, Tokyo 113, Japan
}

\begin{abstract}
We discuss topological aspects of electronic properties of graphene, including edge effects, with the tight-binding model on a honeycomb lattice and its extensions to show the following: (i) Appearance of the pair of massless Dirac dispersions, which is the origin of anomalous properties including a peculiar quantum Hall effect (QHE), is not accidental to honeycomb, but is rather generic for a class of two-dimensional lattices that interpolate between square and $\pi$-flux lattices. Persistence of the peculiar QHE is interpreted as a topological stability. (ii) While we have the massless Dirac dispersion only around $E=0$, the anomalous QHE associated with the Dirac cone unexpectedly persists for a wide range of the chemical potential. The range is bounded by van Hove singularities, at which we predict a transition to the ordinary fermion behavior accompanied by huge jumps in the QHE with a sign change. (iii) For edges we establish a coincidence between the quantum Hall effect in the bulk and the quantum Hall effect for the edge states, which is a manifestation of the topological bulk-edge correspondence. We have also explicitly shown that the $E=0$ edge states in honeycomb in zero magnetic field persist in magnetic field.
\end{abstract}

\section{Introduction}

One fascinating aspect of the condensed-matter physics is that we can have various field theories effectively realized on low-energy scales. Electronic structure in a honeycomb lattice is of particular interest, since the system has zero-mass Dirac cone dispersion at Brillouin zone corners, so we have got an intriguing mixture of a continuum limit and the lattice effect. Graphene, a single layer carbon atoms, provides a prototypical realization of characteristic electronic structures of a honeycomb lattice. This provides interesting problems in condensed matter physics especially in its topological aspects, which is the subject-matter of the present article. Its band dispersion is composed of a pair of $k$-linear bands that touch with each other around $E=0$, so that the graphene is a condensed-matter realization of zero-mass Dirac fermions around $E=0$. Now, the Dirac particles have been known to provide topologically non-trivial structures 12. Physics on the honeycomb lattice thus provides potential links between the field-theoretic concepts and various condensed matter phenomena [3/5].

Indeed, recent experiments have revealed a peculiar type of quantum Hall effect $6 / 7 / 8$. Graphite itself has been studied extensively from many different points of view. There has also been a substantial amount of development as basic materials in nano-technologies 910, where a special interest is characteristic boundary magnetic properties which originate from edge states 1112 . The fact that the honeycomb lattice as interesting edge properties as well as interesting bulk properties is no accident, but this is in fact a realization of hidden physical structures as a topological order 13 14 15. 


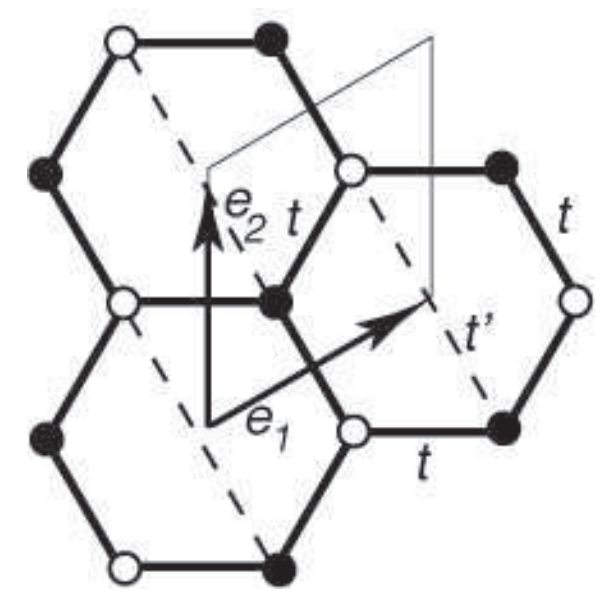

Fig. 1. A honeycomb lattice with a unit cell and the diagonal transfer $t^{\prime}$ indicated.

Topologically non-trivial ground states are, unlike ordinary symmetry-broken states, characterized by geometrical phases rather than by local order parameters. Geometrical phases which are fundamental in quantum physics provide basic tools for characterizing topological orders 1415]16. Appearance of the edge states is one of the important physical consequences of the non-trivial topological orders 17/18/19/20|21. Physical properties of the bulk go hand in hand with those of the edge in the topologically ordered states. So topological properties, which may not so apparent in the bulk, may emerge around the edges or defects with physical consequences. [17/18 19/20]. This bulk-edge correspondence is shared by various topologically ordered states, where a typical one is the quantized Hall (QH) effects [19|20]. Among various topologically ordered states, edge states of the Dirac particle is interesting, especially when the particle is massless 22 23]. This is in fact directly related with the edge states in graphene. To be more precise, honeycomb has a bipartite lattice structure (i.e., a chiral symmetry), which has a fundamental importance in the existence of the $E=0$ edge states. From the topological point of view, appearance of the magnetic moments in the edge states in graphene is understood topologically as a chiral symmetry breaking in edge states 2425.

In this article, we discuss results obtained in the reference [26] focusing on the topological aspects of graphene.

\section{Topological Stability of Dirac Fermions}

We consider a tight-binding Hamiltonian, $H$, for a honeycomb lattice with nearest-neighbor hopping $H^{\prime}$

$$
\begin{gathered}
H=H_{\text {honeycomb }}+H^{\prime} \\
H_{\text {honeycomb }}=t \sum_{\boldsymbol{j}}\left[c_{\bullet}^{\dagger}(\boldsymbol{j}) c_{\circ}(\boldsymbol{j})+e^{i 2 \pi \phi j_{1}} c_{\bullet}^{\dagger}(\boldsymbol{j}) c_{\circ}\left(\boldsymbol{j}-\boldsymbol{e}_{2}\right)+c_{\bullet}^{\dagger}\left(\boldsymbol{j}+\boldsymbol{e}_{1}\right) c_{\circ}(\boldsymbol{j})\right]+\text { h.c. } \\
H^{\prime}=t^{\prime} \sum_{\boldsymbol{j}} e^{-i 2 \pi \phi\left(j_{1}+1 / 2\right)} c_{\bullet}^{\dagger}\left(\boldsymbol{j}+\boldsymbol{e}_{1}-\boldsymbol{e}_{2}\right) c_{\circ}(\boldsymbol{j})+\text { h.c. }
\end{gathered}
$$

where $t=-1$ is the transfer energy (taken to be the unit of energy). Since the honeycomb, a non-Bravais lattice with two sites per unit cell is bipartite, we have defined two kinds of fermion operators, $c_{\circ}(\boldsymbol{j})$ and $c_{\bullet}(\boldsymbol{j})$ as in Fig. 1, where $\boldsymbol{j}=j_{1} \boldsymbol{e}_{1}+j_{2} \boldsymbol{e}_{2}$ labels the unit cell with two translation vectors $\boldsymbol{e}_{1}=(3 / 2, \sqrt{3} / 2) a$ and $\boldsymbol{e}_{2}=(0, \sqrt{3}) a$. 


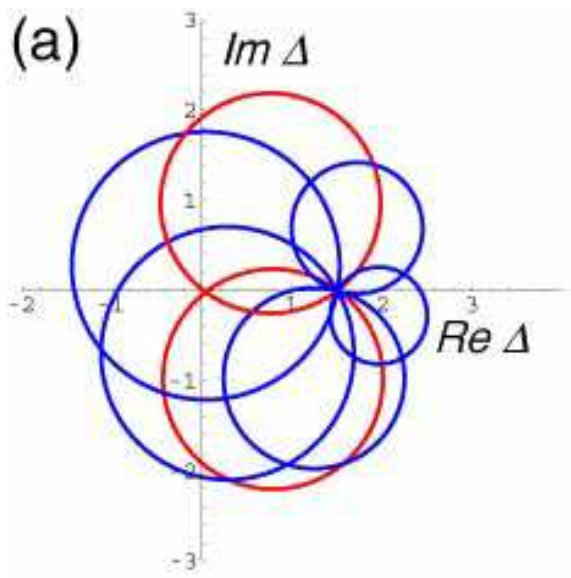

(b)

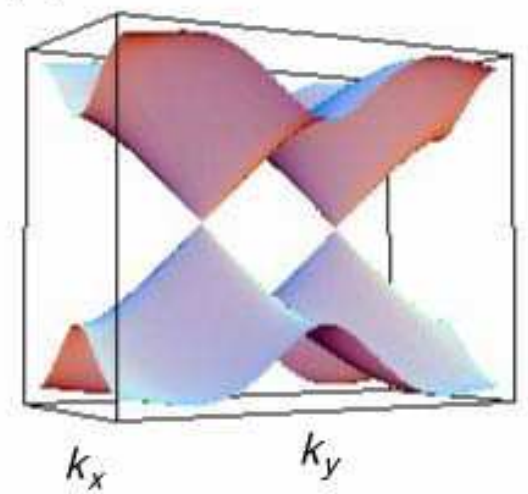

Fig. 2. (a) Locus (each circle) of $\Delta(\boldsymbol{k})$ is shown on the complex $\Delta$-plane when we vary $k_{x}: 0 \rightarrow 2 \pi$ for several fixed values of $k_{y}$ for $t^{\prime} / t=-1 / 2$. The red circle traverses the origin, which implies the appearance of the gapless Dirac fermion. The doubling of the Dirac fermion is also clear from this geometric consideration. (b) Corresponding energy dispersion for $t^{\prime} / t=-1 / 2$.

We then apply an external magnetic field, which is characterized by

$$
\phi=B S_{6} /(2 \pi) \equiv p / q,
$$

the magnetic flux penetrating each hexagon of area $S_{6}=(3 \sqrt{3} / 2) a^{2}$.

Let us first discuss the energy dispersion in zero magnetic field. As is well known, the spectrum of the Hamiltonian $H_{\text {honeycomb }}$ consists of two bands touching with each other at two points (K and $\mathrm{K}^{\prime}$ ) in the Brillouin zone, which are understood as doubled Dirac fermions. Around the zero-gap points the energy dispersion is generically linear in $\left(k_{x}, k_{y}\right)$ space.

Although the appearance of the massless Dirac fermions may seem just an accident for the honeycomb lattice, we shall show here that the appearance of massless Dirac fermions is, surprisingly enough, a generic property and stable against e.g. introduction of a diagonal hopping $H^{\prime}$ if we preserve the chiral symmetry (bipartite lattice with every transfer between A and $\mathrm{B}$ sites). In momentum representation, the Hamiltonian in zero magnetic field is given as

$$
\begin{gathered}
H=\int \frac{d^{2} k}{(2 \pi)^{2}} \boldsymbol{c}^{\dagger}(\boldsymbol{k}) \boldsymbol{h}(\boldsymbol{k}) \boldsymbol{c}(\boldsymbol{k}) \\
\boldsymbol{c}(\boldsymbol{k})=\left(\begin{array}{c}
c_{\bullet}(\boldsymbol{k}) \\
\boldsymbol{c}_{\circ}(\boldsymbol{k})
\end{array}\right), \\
\boldsymbol{h}(\boldsymbol{k})=t\left(\begin{array}{cc}
0 & \Delta(\boldsymbol{k}) \\
\Delta^{*}(\boldsymbol{k}) & 0
\end{array}\right), \\
\Delta(\boldsymbol{k})=1+e^{-i k_{2}}+e^{-i k_{1}}\left[1+\left(t^{\prime} / t\right) e^{i k_{2}}\right] .
\end{gathered}
$$

The chiral symmetry is expressed as

$$
\{\boldsymbol{h}, \boldsymbol{\gamma}\}=0, \quad \gamma=\boldsymbol{\sigma}_{z}=\left(\begin{array}{cc}
1 & 0 \\
0 & -1
\end{array}\right)
$$

which implies that the energy bands are symmetric about $E=0$. For the honeycomb lattice $\Delta$ on the complex plane delineates a circle centered at $C_{0}=1+e^{i k_{2}}$ with a radius $r=$ $\sqrt{1+2\left(t^{\prime} / t\right) \cos k_{2}+\left(t^{\prime} / t\right)^{2}}$ when $k_{1}$ is varied from 0 to $2 \pi$ for a fixed value of $k_{y}$ (Fig.2). By examining $C_{0}$ and $r$, we can show that we have a Dirac cone when the circle goes through the origin, which occurs for $-3 \leq t^{\prime} / t<1$. This shows a topological stability for the appearance of 
the Dirac fermions. That is, Dirac cone persists when the lattice deviates from the honeycomb, even up to the limit of the square lattice where $t^{\prime} / t=1$ in the present notation, unless the chiral symmetry is not broken. We can extend the present line of consideration to the general condition for the appearance of Dirac cones, which will be published elsewhere 27.

\section{Hall conductance of the graphene as the Chern number}

The Hall conductivity of the noninteracting 2D electron systems, as described with the linear response theory, 28] may be regarded as a topological quantum number. 29] Namely, when the Fermi energy lies in the $n$-th gap, the Hall conductivity is given by

$$
\sigma_{x y}=-\frac{e^{2}}{h} c_{\mathrm{F}}\left(E_{\mathrm{F}}\right),
$$

where $c_{\mathrm{F}}$ is an integer called the Chern number, which describes how the wave function responds to a vector potential generated by Berry's gauge potential in the Brillouin zone. If we adopt a Bloch function in the $j$-th energy band $\boldsymbol{\varphi}_{j}(\boldsymbol{k})$ assuming that the energy gap opens over the entire Brillouin zone at each gap in the sequence $\epsilon_{1}(\boldsymbol{k}) \leq \cdots \leq \epsilon_{n}(\boldsymbol{k})<E_{\mathrm{F}}<\epsilon_{n+1}(\boldsymbol{k}) \leq \cdots \leq \epsilon_{2 q}(\boldsymbol{k})$. We can then define a non-Abelian Berry's gauge potential,

$$
\begin{aligned}
& \boldsymbol{A}=\boldsymbol{\Psi}^{\dagger} d \boldsymbol{\Psi} \\
& \boldsymbol{\Psi}=\left(\boldsymbol{\varphi}_{1}, \cdots, \boldsymbol{\varphi}_{n}\right) .
\end{aligned}
$$

Here $A(\boldsymbol{k})$ is an $n \times n$ matrix-valued one-form, which is a difference from the ordinary, scalar Berry's gauge potential. Then the Chern number is given as the $\mathrm{U}(1)$ part of the $\mathrm{U}(n)$ gauge potential,

$$
c_{\mathrm{F}}\left(E_{\mathrm{F}}\right)=\frac{1}{2 \pi i} \int \operatorname{Tr} d A .
$$

This formulation, a non-Abelian extension of Berry's gauge potential, 14 15 may seem too elaborate, but is useful when there are multiple bands below the Fermi energy. Namely, this formula holds even if some gaps in the Fermi sea are closed, as long as the bands in question, $\epsilon_{n}(\boldsymbol{k})$ and $\epsilon_{n+1}(\boldsymbol{k})$, do not overlap. In the special case when all the bands in the Fermi sea are separated with each other, the Chern number, Eq.(1), is simply the sum of the Chern numbers assigned to individual bands.

To evaluate the expression, here we have employed a technique developed recently in the lattice gauge theory by two of the present authors 30131. The numerical results for the Chern number $c_{\mathrm{F}}$, i.e., the Hall conductivity per spin, as a function of the chemical potential $E_{\mathrm{F}}$ for honeycomb $\left(t^{\prime}=0\right)$ is shown in Fig.3. While the integral Chern number $c_{\mathrm{F}}$ is defined only for each gap, we have plotted $c_{\mathrm{F}}$ as step functions, which makes sense as long as the magnetic field is not too large, as in Fig. 3 with $\phi=1 / 31$, since the width of each Landau band is then much smaller than the size of gaps.

The result shows a striking answer to a key question: what is the fate of the Dirac-like behavior as we go away from $E=0$. When the Fermi energy is swept in the honeycomb lattice, the Dirac-like Hall conductivity with steps of two (or four when spin degeneracy is included) in units of $-e^{2} / h$ exist around $E=0$ as has been noted by many authors. 6778 32 Let us call this Dirac-Landau behavior. Now, we can see that this Dirac-Landau behavior persists all the way up to some energy, $E_{v H}=|t|$, which we can identify to be the van Hove singularities appearing in Fig. 3. We can notice that huge steps accompanied by a sign change in the Hall conductivity occurs at these points. This result implies the following: The effective theory near the zero energy is Dirac-like fermions in the continuum limit, for which an unconventional quantization of the Hall conductivity has been derived in the continuum limit from the Dirac Landau levels. 69. The present calculation leads to the conclusion that the unusual property extends to an unexpectedly wide region of energy in the actual lattice fermion system. 


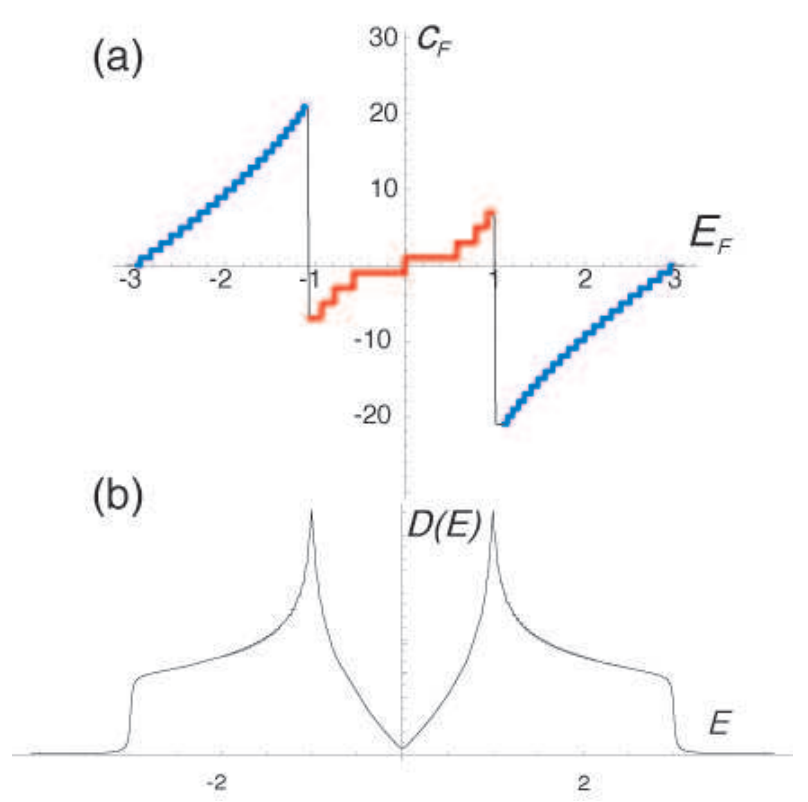

Fig. 3. (a) The Chern number (Hall conductivity per spin in units of $-e^{2} / h$ ) for magnetic field $\phi=1 / 31$ is plotted against the Fermi energy $E_{\mathrm{F}}$ for $t^{\prime} / t=0$. The red lines indicate the steps of two in the Chern number sequence $\left(\sigma_{x y}= \pm(2 N+1) e^{2} / h, N\right.$ : integer $)$, while the blue lines step of one $\left(\sigma_{x y}= \pm N e^{2} / h\right)$. (b) Density of states for the honeycomb lattice.

Outside the van Hove energies (i.e., in the band-edge regions), we recover the conventional quantum Hall effect (QHE) where the step changes by one in units of $-e^{2} / h$ (which we call Fermi-Landau). This automatically implies that a huge step accompanied by a sign change has to occur at the boundary between Dirac-Landau and Fermi-Landau behaviors. In other words the bands situated at the van-Hove singularities induce a change, which is topological in that the relevant quantum numbers are topological. The Hall conductivity per spin on the honeycomb lattice for the entire energy region is summarized as $(N=0,1,2, \cdots)$

$$
\sigma_{x y}=-\frac{e^{2}}{h} \times \begin{cases}+(N+1) \quad E_{\mathrm{F}}<-E_{v H}, & : \text { Landau index from the band bottom } \\ -(2 N+1)-E_{v H}<E_{\mathrm{F}}<0, & : \text { Dirac-Landau index from the zero energy } \\ +(2 N+1) 0<E_{\mathrm{F}}<+E_{v H}, & : \text { Dirac-Landau index from the zero energy } \\ -(N+1) \quad E_{v H}<E_{\mathrm{F}}, & : \text { Landau index from the band top }\end{cases}
$$

We should again double these numbers if we include the spin degeneracy. While the unconventional quantization around the zero energy has been beautifully observed experimentally $7 / 8$, pushing the chemical potential further to approach the van Hove energies should detect the topological phase transition.

\section{Bulk-Edge Correspondence in Graphene: Edge states of the Dirac Fermions}

When a system has a nontrivial topological structure, edge states should generically exhibit characteristic properties. For the honeycomb lattice, edge states and their flat dispersion have been intensively discussed with its relevance to spin alignment[11/12. Another way of saying is that the honeycomb system has a bipartite symmetry, which guarantees the presence of the zero mode edge states 24], i.e., there exist macroscopic edge states at the zero energy unless the bipartite symmetry is broken. This gives rise to dispersionless edge states. The flat bands are unstable against bipartite symmetry breaking (Peierls instability), which is, e.g., realized 
(a)

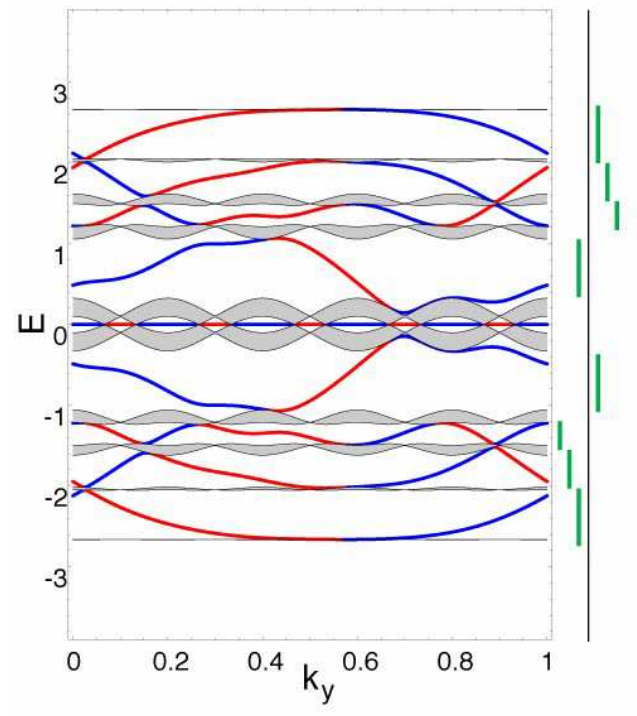

(b)

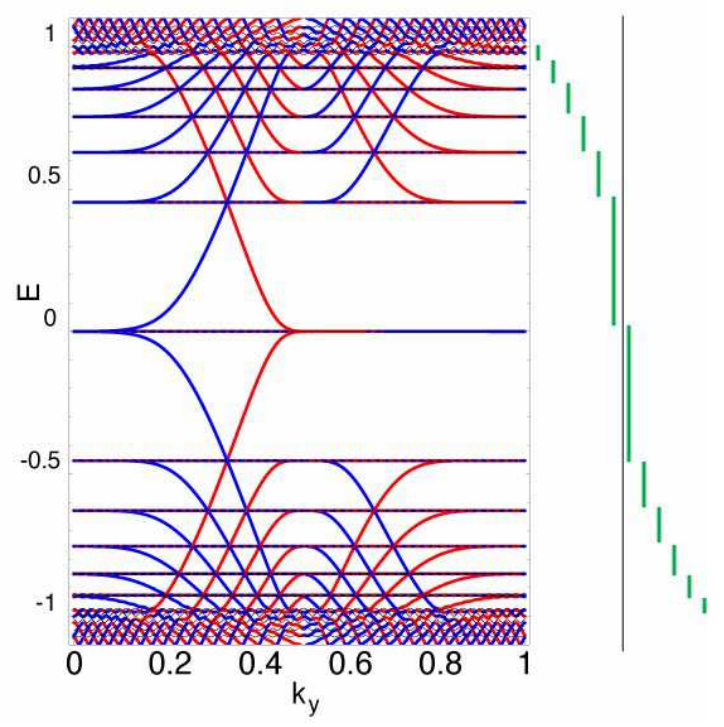

Fig. 4. The energy spectra for the cylindrical system in Fig. 1 with the flux $\phi=1 / 5$ for $t^{\prime} / t=$ 0 (honeycomb). $k_{y}$ is a momentum along the cylindrical direction. Blue lines represent edge states localized at the right (bearded) edge, while red ones the left (zigzag) edge, with the bulk energy bands shown as grey regions.

when an antiferromagnetic spin ordering occurs. This may be viewed as a topological origin of the boundary spin moments. [25]

Now, the problem of edge states vs bulk states is particularly interesting for the QHE, since the problem of how the Dirac-Landau QHE $\propto(2 N+1)$ is related to the edge states is fundamentally interesting. One of the present authors has shown, for 2D square (or anisotropic square) systems, that the edge states whose energy dispersions run across the Landau bands have topological QHE numbers, for which $\sigma_{x y}^{\text {edge }}=\sigma_{x y}^{\text {bulk }}$ by identifying the connection between the topological integers for the bulk and for the edge states. 20121. So let us now focus on the edge states of the honeycomb lattice in terms of the topological structure.

We follow the analysis in refs 1920121 to reduce the system to a one-dimensional model by a partial Fourier transform along the second direction $\left(j_{2}\right)$ of the fermion operators, $c_{\circ}, \bullet(j)=$ $L_{2}^{1 / 2} \sum_{k_{2}} e^{i k_{2} j_{2}} c_{\circ}, \bullet\left(j_{1}, k_{2}\right)$. This yields a $k_{2}$-dependent series of one-dimensional Hamiltonian

$$
\begin{aligned}
& H=\sum_{k_{2}} H_{1 \mathrm{D}}\left(k_{2}\right) \\
& H_{1 \mathrm{D}}\left(k_{2}\right)=\sum_{j_{1}}\left[t_{\bullet}\left(j_{1}, k_{2}\right) c_{\circ}^{\dagger}\left(j_{1}, k_{2}\right) c_{\bullet}\left(j_{1}, k_{2}\right)+t_{\bullet}\left(j_{1}, k_{2}\right) c_{\bullet}^{\dagger}\left(j_{1}+1, k_{2}\right) c_{\circ}\left(j_{1}, k_{2}\right)\right]+\text { h.c. }
\end{aligned}
$$

where $k_{2}$-dependent hopping parameters are

$$
\begin{aligned}
& t_{\circ \bullet}\left(j_{1}, k_{2}\right)=t\left(1+e^{i k_{2}-i 2 \pi \phi j_{1}}\right), \\
& t_{\bullet \circ}\left(j_{1}, k_{2}\right)=t\left[1+\left(t^{\prime} / t\right) e^{i k_{2}-i 2 \pi \phi\left(j_{1}+1 / 2\right)}\right] .
\end{aligned}
$$

We consider cylindrical systems with a zigzag edge at one end and a bearded (or Klein's) edge at the other. We have adopted this geometry, since zigzag-bearded system has an $E=0$ edge state in zero magnetic field that extend over the entire Brillouin zone, as first pointed out in [33]. 
(a)

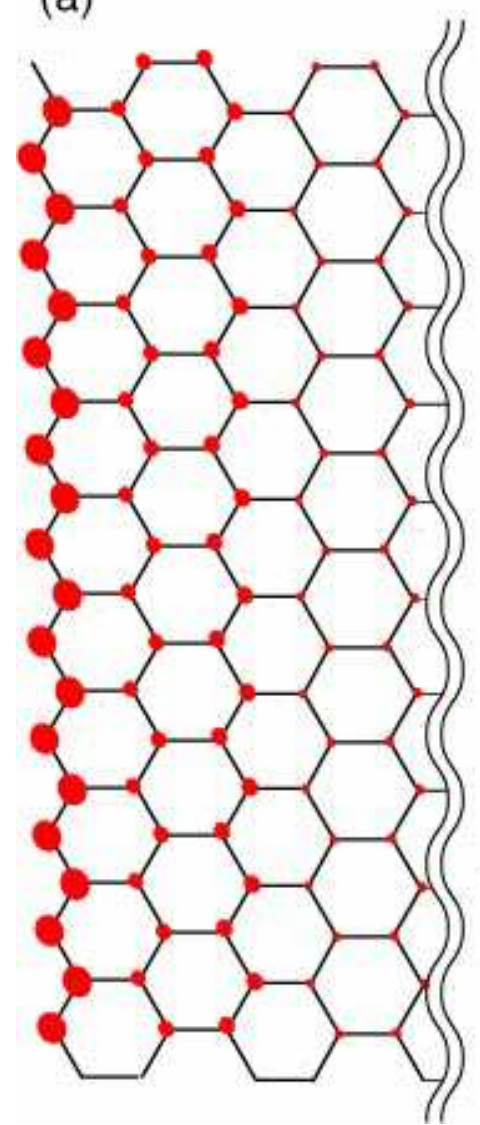

(b)

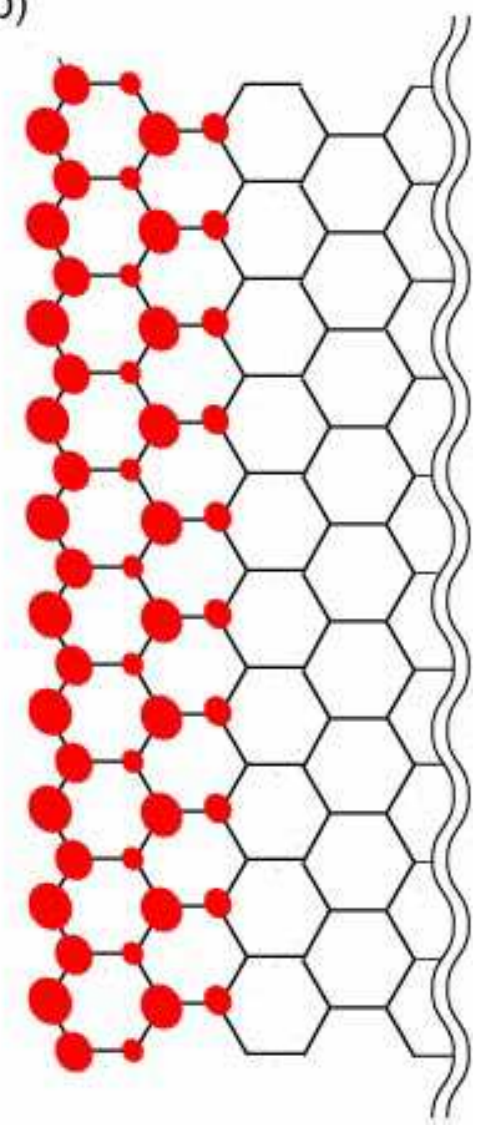

Fig. 5. The charge density of the edge states at the zigzag edge for $\phi=1 / 3$. (a) $E=-0.95$ and $E=-1.5$.

A remarkable feature in the spectrum shown here is that, on top of the edge states across adjacent Landau bands, there always exist exactly $E=0$ edge states with a flat dispersion, which has to exist since the number of the edge states are odd, $2 q-1$. Namely, a system with zigzag edges has a zero-energy edge mode in some region of $k_{2}$, a system with bearded edges in the other region, and the present system has the mode over the entire region. This is a simple extension of the discussion in the absence of a magnetic field. 24,25]

With Laughlin's argument [17, supplemented by the behavior of the edge state (as a function of the momentum $k_{2}$, , one can identify a topological number $I\left(E_{\mathrm{F}}\right)$, the number of electrons carried by Laughlin's adiabatic procedure, for each edge branch when the chemical potential $E_{\mathrm{F}}$ traverses the branch. [17192021] As shown in Fig.4, we can readily confirmed a bulk-edge correspondence,

$$
\begin{aligned}
\mathrm{c}_{\mathrm{F}}\left(E_{\mathrm{F}}\right) & =I\left(E_{\mathrm{F}}\right) \\
\sigma_{x y}^{\text {bulk }} & =\sigma_{x y}^{\text {edge }}
\end{aligned}
$$

for Dirac fermions in graphene.

As for the edge states, we display in Fig. 5 typical wave functions in real space. We can see that each of the left and right edge states decays into the bulk, where the decay length depends on whether its energy is close to the touching of the edge mode and the Landau level. 


\section{5 summary}

In summary, we have shown that the Landau levels are divided by the van Hove singularities into two regimes: One is effectively described by Dirac particles, and the other by ordinary finite-mass fermions. We have confirmed this both from the Chern number argument and by the bulk-edge correspondence. Topological considerations are useful throughout.

The properties revealed here for the whole spectrum of the honeycomb lattice may be experimentally observable if the chemical potential can be varied over a wide range. In real graphene samples there may be disorder, in which case we are talking about a dirty Hofstadter system. However, non-monotonic behaviors should survive the disorder as far as the degree of disorder is not too large, as has been indicated by a numerical calculation for a dirty Hofstadter system 34 .

\section{References}

1. G. Semenoff, Phys. Rev. Lett. 53, 2449 (1984).

2. F. D. M. Haldane, Phys. Rev. Lett. 61, 2015 (1987).

3. C. L. Kane and E. J. Mele, Phys. Rev. Lett. 95, 146802 (2005)

4. A. W. W. Ludwig, M. P. A. Fisher, R. Shankar, and G. Grinstein, Phys. Rev. B 50, 7526 (1993).

5. Y. Hatsugai and M. Kohmoto, Phys. Rev. B 42, 8282 (1990).

6. V. P. Gusynin and S. Sharapov, Phys. Rev. Lett. 95, 146801 (2005).

7. K. S. Novoselov, Nature, 438, 197 (2005); Nature Physics, 2, 177 (2006).

8. Y. Zhang, Y.-W. Tan, H. L. Stormer, and P. Kim, Nature, 438, 201 (2005).

9. Y. Zheng and T. Ando, Phys. Rev. B 65, 245420 (2002).

10. T. Ando, J. Phys. Soc. Jpn. 74, 777 (2005).

11. M. Fujita, K. Wakabayashi, K. Nakada, and K. Kusakabe, J. Phys. Soc. Jpn. 65, 1920 (1996).

12. K. Wakabayashi, M. Fujita, H. Ajiki, and M. Sigrist, Phys. Rev. B 59, 8271 (1999).

13. X. G. Wen, Phys. Rev. B 40, 7387 (1989).

14. Y. Hatsugai, J. Phys. Soc. Jpn. 73, 2604 (2004a).

15. Y. Hatsugai, J. Phys. Soc. Jpn. 74, 1374 (2004b).

16. A. Shapere and F. Wilczek, eds., Geometric Phases in Physics (World Scientific, 1989).

17. R. B. Laughlin, Phys. Rev. B 23, 5632 (1981).

18. B. I. Halperin, Phys. Rev. B 25, 2185 (1982).

19. Y. Hatsugai, Phys. Rev. B 48, 11851 (1993a).

20. Y. Hatsugai, Phys. Rev. Lett. 71, 3697 (1993b).

21. Y. Hatsugai, J. Phys. Condens. Matter 9, 2507 (1997).

22. E. Witten, Phys. Lett. 117B, 324 (1982).

23. A. J. Niemi and G. Semenoff, Phys. Rev. Lett. 51, 2077 (1983).

24. S. Ryu and Y. Hatsugai, Phys. Rev. Lett. 89, 077002 (2002).

25. S. Ryu and Y. Hatsugai, Physica C 388-389, 90 (2003).

26. Y. Hatsugai, T. Fukui and H. Aoki, Phys. Rev. B 74, 205414 (2006).

27. Y. Hatsugai, T. Fukui and H. Aoki, unpublished.

28. H. Aoki and T. Ando, Solid State Comm. 38, 1079 (1981).

29. D. J. Thouless, M. Kohmoto, P. Nightingale, and M. den Nijs, Phys. Rev. Lett. 49, 405 (1982).

30. T. Fukui, Y. Hatsugai, and H. Suzuki, J. Phys. Soc. Jpn. 74, 1674 (2005).

31. Y. Hatsugai, T. Fukui, and H. Suzuki, cond-mat/0507466 (2005).

32. D. N. Sheng, L. Sheng, and Z. Y. Weng, cond-mat/0602190.

33. K. Kusakabe and Y. Takagi, Mol. Cryst. Liq. Cryst. 387, 7 (2002).

34. H. Aoki, Surf. Sci. 263, 137 (1992). 